\title{
Multimorbilidade e comorbilidade: duas perspectivas da mesma realidade
}

Paula Broeiro*

A presença de mais de um problema de saúde em cada pessoa é uma constante e uma preocupação em cuidados de saúde primários. A designada multimorbilidade tem sido estudada de forma heterogénea, desde a simples contagem do número de doenças por indivíduo a sistemas sofisticados de classificação e medição da carga de doença. Contudo, a evidência sobre o benefício das intervenções é limitada. ${ }^{1-2}$ Torna-se premente a caracterização de padrões e a identificação de intervenções e metas que deverão ser iniciadas por uma clara definição do conceito multimorbilidade: dois ou mais problemas de saúde diagnosticados na mesma pessoa, não definindo nenhum como central. ${ }^{1,3-4}$

O termo 'multimorbilidade' surgiu como uma alternativa "democrática" ao termo "comorbilidade". ${ }^{5}$ Este último designa "qualquer problema independente $\mathrm{e}$ adicional a uma doença existente e em estudo" - doença ou problema índice -, colocando-a em posição central e as restantes em situação secundária, interferindo ou não no curso e tratamento da doença índice. ${ }^{5-6} \mathrm{~A}$ distinção entre multimorbilidade e comorbilidade parece, assim, fictícia, dependendo da perspectiva de análise, uma vez que o mesmo paciente tem multimorbilidade para o seu Médico de Família e comorbilidade para o seu Pneumologista (e.g., Asma, doença índice).

Ambos os termos, "comorbilidade" e "multimorbilidade", se referem a múltiplos problemas ou condições de saúde, sem todavia ser clara a distinção entre o que é considerado como doença ou como factor de risco (e.g., Hipertensão é uma doença ou um factor de risco?). ${ }^{5}$ Esta indefinição constitui uma limitação, presente

*Directora da Revista Portuguesa de Medicina Geral e Familiar em muitos dos estudos sobre esta temática, mas outras relacionam-se com a não identificação de gravidade das doenças e suas repercussões na vida, bem como com a não inclusão da prioridade do paciente em paralelo com a do médico (e.g., insuficiência cardíaca médico $v s$ osteoartrose-paciente). ${ }^{5}$

A abordagem de múltiplos problemas e condições de saúde crónicos de uma única pessoa, na perspectiva de um problema índice e comorbilidades, torna-se ineficaz e ineficiente, pois considera a maioria dos problemas apenas na sua relação com o problema índice. ${ }^{6}$ Em sentido inverso, o conceito de multimorbilidade potencia a abordagem da totalidade dos problemas (holística), tendo em conta o sentir da pessoa (centrada no paciente) e promove a compreensão da interacção entre problemas físicos, mentais e sociais (complexidade). ${ }^{6}$

Não dispondo de dados recentes de prevalência em Portugal socorremo-nos de uma revisão sistemática de estudos de prevalência em contexto de cuidados de saúde primários, cujos resultados revelaram estimativas globais que oscilaram entre $12,9 \%$ na população adulta e 95,1\% na população com 65 anos ou mais. ${ }^{1}$ Ressalta desses estudos que a multimorbilidade se encontra consistentemente associada à idade (igual ou superior a 65 anos), ao género feminino, ao baixo nível socioeconómico, ${ }^{1-2}$ bem como à doença mental. ${ }^{1}$

É, pois, consensual que o envelhecimento populacional aumenta a carga de doença crónica e a prevalência de doentes com multimorbilidade. ${ }^{3-4,7-8} \mathrm{Na}$ população de mais idade, a multimorbilidade é a regra e não a excepção. Contudo, o impacto da multimorbilidade na dependência está por esclarecer. ${ }^{9}$ Koller et al desenvolveram um estudo de coorte com a duração de cinco anos (2005 a 2009), numa população idosa, com 
o objectivo de estimar o impacto da multimorbilidade na dependência e necessidade de cuidados de longo prazo. A média de seguimento foi de 4,5 anos e os resultados confirmam, apenas, que as pessoas com multimorbilidade tiveram um maior risco de se tornarem dependentes de cuidados (HR: 1,85; IC 1,78-1,92). ${ }^{7}$

Os doentes com multimorbilidade (ou com elevado número de comorbilidades, quando está em estudo um problema-índice) são geralmente excluídos da investigação clínica, pelo que a natureza e a complexidade das necessidades destes doentes carecem de melhor compreensão e suporte de evidência. ${ }^{2,9}$ Estudos de coorte prospectivos são um método robusto de descrever e estudar a história natural das condições de saúde crónicas, bem como os efeitos da multimorbilidade ao longo do tempo e os factores que influenciam estes efeitos. Sem o conhecimento gerado por esse tipo de estudos não será possível partir para o desenvolvimento de intervenções eficazes. ${ }^{9}$

A multimorbilidade coloca em questão o paradigma de gestão da doença (definida, desenvolvida e orientada para a doença), a investigação e a educação médica tradicionais. Numa revisão sistemática de estudos qualitativos de Sinnott et al, publicada no BMJ em 2013, são sintetizados os quatro principais constrangimentos ao cuidar de doentes com multimorbilidade: desorganização e fragmentação dos cuidados de saúde; inadequação das normas de orientação clínica e da Medicina baseada em evidência; desafios da prestação de cuidados centrados no paciente e barreiras no processo de tomada de decisão partilhada. ${ }^{10}$

A dissonância de conceitos, princípios e interesses, como a organização dos sistemas de saúde, a especialização médica ou a distinção entre curar e cuidar geram a desorganização e fragmentação dos cuidados de saúde e dificultam a abordagem da multimorbilidade. As diferentes políticas têm afastado o Médico de Família do seu core business, a abordagem holística do paciente, neste caso particular, da multimorbilidade, fazendo-o sentir-se, por vezes, excluído do cuidado. ${ }^{10}$

O método clínico centrado no paciente ${ }^{11}$ é essencial quando se considera que um sistema de saúde deve cuidar de pessoas com multimorbilidade. ${ }^{6}$ Não existindo um problema dominante, o cuidado centrado no paciente é o ideal para equilibrar todos os problemas e permitir uma abordagem integrada. ${ }^{6}$ A medição de desempenho para a multimorbilidade não foi adequadamente desenvolvida, importando salientar que os indicadores específicos de doença (e.g., diabetes) podem ser atingidos, mas desadequados ou descontextualizados, em pacientes com multimorbilidade. ${ }^{6}$

Os objectivos do cuidado aos pacientes com multimorbilidade são evitar a iatrogenia, reduzir a morbilidade e mortalidade, sem comprometer a funcionalidade e qualidade de vida. Mas os instrumentos existentes não contemplam estes objectivos e não incluem as necessidades e a complexidade do cuidar destes pacientes com multimorbilidade. ${ }^{6}$

Em muitos países foi implementado o modelo de gestão integrada da doença, que mantém a lógica doença única, integrando o doente na organização através do gestor de caso, mas não o cuidado ao paciente. ${ }^{12} \mathrm{Os}$ pacientes com multimorbilidade estão ligados a vários médicos e serviços, não coordenados entre si, e ficam confusos quanto a quem é responsável e por quê, podendo existir problemas insuficientemente controlados e outros duplamente tratados. ${ }^{12}$ As necessidades dos pacientes com multimorbilidade não são a soma das necessidades decorrentes das doenças individuais e, portanto, a organização por doença única tem um efeito negativo na continuidade dos cuidados. ${ }^{12}$

A complexidade ligada à multimorbilidade acarreta dificuldades na gestão dos cuidados de saúde e das terapêuticas ${ }^{9,13}$ e pode assumir diferentes níveis: individual (paciente), organizacional (sistema de saúde) e profissional (médico). ${ }^{12}$ Uma melhoria dos cuidados prestados a doentes com multimorbilidade será um desafio para todos os sistemas de saúde, exigindo cuidados de saúde primários fortes que integrem uma abordagem holística, centrada no paciente e na complexidade da multimorbilidade ${ }^{9}$ e um sistema de saúde focado no cuidar, contrariando a lógica de serviços de saúde especializados e fragmentados. ${ }^{14} \mathrm{~A}$ colaboração eficiente entre profissionais e serviços é essencial, devendo os responsáveis valorizar os seus Médicos de Família e priorizar a continuidade dos cuidados e a melhoria da qualidade de vida e funcionalidade dos pacientes, ${ }^{15}$ ao invés de premiar o mero atingimento de indicadores de doença descontextualizados. ${ }^{14}$

\section{REFERÊNCIAS BIBLIOGRÁFICAS}

1. Violan C, Foguet-Boreu Q, Flores-Mateo G, Salisbury C, Blom J, Freitag 
$M$, et al. Prevalence, determinants and patterns of multimorbidity in primary care: a systematic review of observational studies. PLoS ONE. 2014;9(7):e102149.

2. Smith SM, Soubhi H, Fortin M, Hudon C, O'Dowd T. Managing patients with multimorbidity: systematic review of interventions in primary care and community settings. BMJ. 2012;345:e5205.

3. France EF, Wyke S, Gunn JM, Mair FS, McLean G, Mercer SW. Multimorbidity in primary care: a systematic review of prospective cohort studies. Br J Gen Pract. 2012;62(597):e297-e307.

4. Barnett K, Mercer SW, Norbury M, Watt G, Wyke S, Guthrie B. Epidemiology of multimorbidity and implications for health care, research, and medical education: a cross-sectional study. The Lancet. 2012;380(9836):37-43.

5. Valderas JM, Mercer SW, Fortin M. Research on patients with multiple health conditions: different constructs, different views, one voice. J Comorbidity. 2011;1(1):1-3.

6. Boyd CM, Fortin M. Future of multimorbidity research: how should understanding of multimorbidity inform health system design? Public Health Rev. 2011;33(2):451-74.

7. Koller D, Schön G, Schäfer I, Glaeske G, van den Bussche H, Hansen H. Multimorbidity and long-term care dependency: a five-year follow-up. BMC Geriatr. 2014;14:70.

8. Laan W, Bleijenberg N, Drubbel I, Numans ME, De Wit NJ, Schuurmans $M J$. Factors associated with increasing functional decline in multimor- bid independently living older people. Maturitas. 2013;75(3):276-81.

9. Mercer SW, Gunn J,Wyke S. Improving the health of people with multimorbidity: the need for prospective cohort studies. J Comorbidity. 2011;1(1):4-7.

10. Sinnott C, Mc Hugh S, Browne J, Bradley C. GPs' perspectives on the management of patients with multimorbidity: systematic review and synthesis of qualitative research. BMJ Open. 2013;3:e003610.

11. Stewart M, Brown J, Levenstein J, McCracken E, McWhinney IR. The patient-centred clinical method. 3. Changes in residents' performance over two months of training. Fam Pract. 1986;3(3):164-7.

12. Doessing A, Burau V. Care coordination of multimorbidity: a scoping study. J Comorbidity. 2015;5(1):15-28.

13. Giovannetti ER, Wolff JL, Xue QL, Weiss CO, Leff B, Boult C, et al. Difficulty assisting with health care tasks among caregivers of multimorbid older adults. J Gen Intern Med. 2012;27(1):37-44.

14. Salisbury C. Multimorbidity: time for action rather than words. Br J Gen Pract. 2013;63(607):64-5.

15. Smith SM, Soubhi H, Fortin M, Hudon C, Dowd OT. Interventions for improving outcomes in patients with multimorbidity in primary care and community settings. Cochrane Database Syst Rev. $2012 \mathrm{Apr}$ 18:4:CD006560.

\section{ENDEREÇO PARA CORRESPONDÊNCIA}

director@rpmgf.pt 